\title{
Speckle Tracking Echocardiography and All-Cause and Cardiovascular Mortality Risk in Chronic Kidney Disease Patients
}

\author{
Laura Jahn $^{\mathrm{a}}$ Rafael Kramann ${ }^{\mathrm{a}} \quad$ Nikolaus Marx $^{\mathrm{b}}$ Jürgen Floege ${ }^{\mathrm{a}}$ \\ Michael Becker ${ }^{\mathrm{b}}$ Georg Schlieper ${ }^{\mathrm{a}, \mathrm{c}}$ \\ a Department of Nephrology and Clinical Immunology, Uniklinik RWTH Aachen, Aachen, \\ Germany; ${ }^{b}$ Department of Cardiology, Uniklinik RWTH Aachen, Aachen, Germany; ${ }^{\circ}$ Center \\ for Nephrology, Hypertension and Metabolic Diseases, Hannover, Germany
}

\section{Keywords}

Chronic kidney disease $\cdot$ Speckle tracking echocardiography · Cardiovascular · Mortality

\begin{abstract}
Background and Objectives: Patients with chronic kidney disease (CKD) exhibit a highly increased risk of cardiovascular (CV) morbidity and mortality. Subtle changes in left ventricular function can be detected by two-dimensional (2D) speckle tracking echocardiography (STE). This study investigated whether myocardial dysfunction detected by 2D STE may aid in CV and all-cause mortality risk assessment in patients with CKD stages 3 and 4. Method: A study group of 285 patients (CKD 3: 193 patients; CKD 4: 92 patients) and a healthy control group (34 participants) were included in the retrospective study. 2D STE values as well as early and late diastolic strain rates were measured in ventricular longitudinal, circumferential and radial directions. Patients' CV and all-cause outcome was determined. Results: In the CKD group all measured longitudinal STE values and radial strain were significantly reduced compared to the control group. Cox proportional hazards regression revealed global longitudinal strain to predict CV and all-cause mortality (hazard ratio [HR] 1.15, 95\% Cl 1.06-1.25; $p=0.0008$ and HR 1.09, 95\% Cl 1.04-1.14; $p=0.0003)$. After adjustment for sex, age, diabetes, estimated glomerular filtration rate, and preexisting CV disease, this association was maintained for CV mortality and all-cause mortality $(\mathrm{HR} 1.16,95 \% \mathrm{Cl} 1.06-1.27 ; p=0.0019$ and $\mathrm{HR} 1.08,95 \% \mathrm{Cl}$ $1.03-1.14 ; p=0.0026$, respectively). Conclusions: The present study shows that 2D STE detects reduced left ventricular myocardial function and allows the prediction of CV and all-cause mortality in patients at CKD stages 3 and 4.




\section{Kidney \\ Blood Pressure \\ Research}

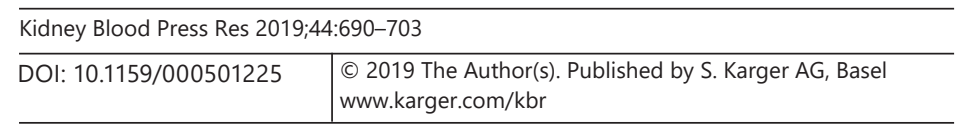

Jahn et al.: Strain and Mortality in CKD Patients

\section{Introduction}

Patients with chronic kidney disease (CKD) exhibit a highly increased risk of cardiovascular (CV) morbidity and mortality compared to the general population. CV disease is considered to be responsible for $50-60 \%$ of all deaths in patients with CKD. Patients with CKD are at a higher risk of dying from $\mathrm{CV}$ disease than reaching end-stage renal disease (ESRD) [1-3]. CKD patients experience angina or myocardial infarction symptoms in an atypical manner, which considerably complicates the diagnosis of $\mathrm{CV}$ disease in this high-risk patient group [4]. It is generally accepted that there are complex mechanisms leading to abnormal cardiac structure and function (confirmed in animals and humans) correlating with increased CV mortality in CKD patients $[3,5,6]$. However, concerns about radiocontrast nephropathy and toxicity of gadolinium-containing contrast agents often limit an adequate work-up of suspected CV disease in CKD patients. Therefore, non-invasive and non-contrast based diagnostic methods that can detect early structural and functional myocardial abnormalities and that can identify patients at risk for CV disease are necessary to initiate adequate diagnostic, preventive, and therapeutic measures.

Two-dimensional (2D) speckle tracking echocardiography (STE) has been developed as a novel way to provide a non-invasive assessment of cardiac function. The method uses natural acoustic markers (speckles) spread throughout the myocardium, which are tracked during the cardiac cycle to follow myocardial movement [7-9]. This information can be processed to determine both myocardial deformation (strain [\%]) and velocity of deformation (strain rate [1/s]) [10].

Translational and tethering effects affect the motion of any myocardial segment [11]. In contrast to conventional echocardiographic methods, STE is able to differentiate passive from active change of location by subtracting the abovementioned effects [8]. Thus, STE allows exact identification of regional segments with restricted contractility (e.g., scar tissue).

Recently, STE has been shown to recognize uremic cardiomyopathy in ESRD and to predict CV mortality [11]. There are several studies confirming the identification of cardiac dysfunction in different cohorts with normal and impaired kidney function by STE [12-16], but only a few focused on the prognostic value, especially in CKD stages 3 and 4 [17-19].

Thus this study investigated whether STE detects reduced cardiac function and whether it allows prediction of CV and all-cause mortality in patients with CKD stage 3 or 4 .

\section{Materials and Methods}

\section{Patient Population}

Between January 1, 2008 and December 31, 2011, 3,779 patients with a diagnosis of CKD at stage 3 or 4 (ICD 10: N18.3 or N18.4) were investigated with echocardiography, at least once in the RWTH Aachen University Hospital Department of Cardiology. Of these, 350 patients were recruited by screening the first 270 patients per each year (sorted by hospitalization date) for inclusion criteria (correct echocardiography machine, required chamber views and short-axis views, laboratory data within 2 weeks before or after echocardiographic examination).

Sixty-five patients had to be excluded due to suboptimal picture quality for strain analysis or re-classification of CKD stage due to serum creatinine. The CKD Epidemiology Collaboration equation was used to estimate the kidney function and estimated glomerular filtration rate (eGFR).

Finally, 285 patients (181 men [64\%]; mean age $71 \pm 11$ years) were included and 2D strain analysis was performed (Fig. 1). 


\section{Kidney \\ Blood Pressure \\ Research}

\begin{tabular}{l|l}
\hline Kidney Blood Press Res 2019;44:690-703 \\
\hline DOI: 10.1159/000501225 & $\begin{array}{l}\text { @ 2019 The Author(s). Published by S. Karger AG, Basel } \\
\text { www.karger.com/kbr }\end{array}$ \\
\hline
\end{tabular}

Jahn et al.: Strain and Mortality in CKD Patients
Fig. 1. Chart of the recruitment of patients. CKD, chronic kidney disease.

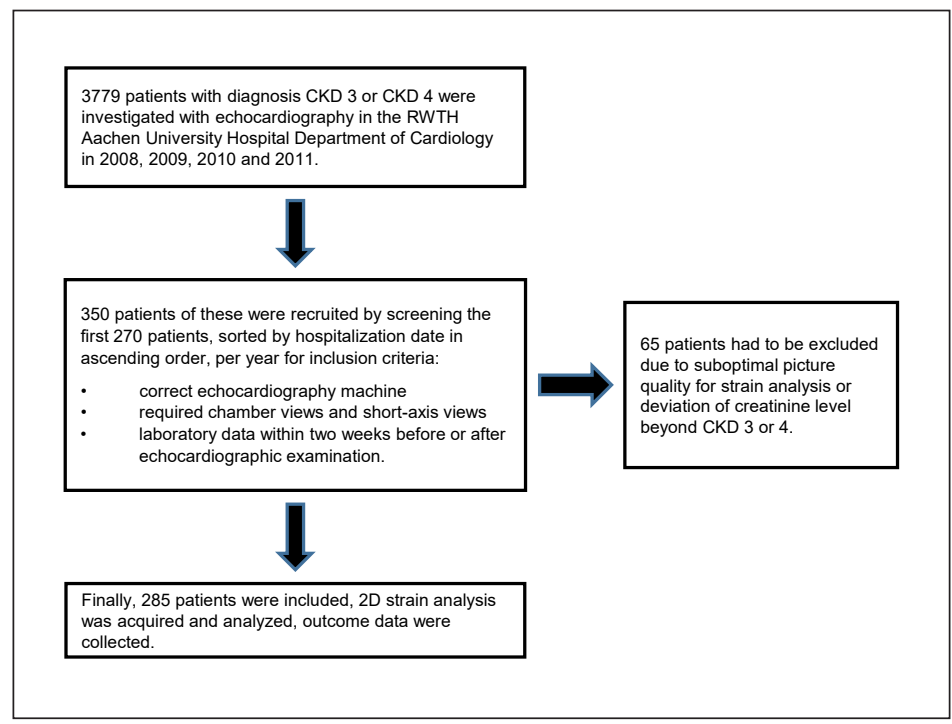

For comparison between strain values of CKD 3-4 patients and healthy persons, an agematched control group of 34 participants without renal or CV disease from a study previously performed at our hospital has been observed (23 men [68\%]; mean age $69 \pm 9$ years, serum creatinine level $<106 \mu \mathrm{mol} / \mathrm{L}$, left ventricular ejection fraction [LVEF] $>55 \%$, Simpson biplane method). Patient information concerning personal data, vitals, medical history, medications, and laboratory data were collected by retrospective chart view.

Two and a half years after the echocardiographic examination, follow-up data were determined. In this regard we registered CV events (coronary artery disease, ST-elevation myocardial infarction [STEMI], non-STEMI [NSTEMI], stroke), bypass surgery or percutaneous transluminal coronary angioplasty, death, and cause of death.

\section{D Echocardiography and Speckle Tracking Analysis}

On the basis of 2D echocardiographs prospectively performed in the unit of the Cardiology Department in our hospital from 2008 to 2011, 2D strain analysis could be performed.

Left ventricular parasternal short-axis view at mid-papillary muscle level such as left ventricular apical 2-, 3-, and 4-chamber views were acquired using a digital ultrasound scanner (Vivid 7; GE Healthcare) provided with a 25-MHz transducer [11].

LVEF was quantified with EchoPAC-PC software version 110.1.3 (General Electric, Horton, Norway) by manual tracing of end-systolic and -diastolic endocardial borders using apical 4- and 2-chamber-views (frame rate: 50-90 FPS) employing the Simpson biplane method. Preserved LVEF was defined as $>50 \%$ [20].

STE analysis was processed retrospectively and offline with the software mentioned above (Fig. 2).

The ventricle performs a complex 3-dimensional motion because of refined myocardial fiber orientation. Three components of myocardial contraction were classified:

Longitudinal: parallel to longitudinal axis, from base to apex

Circumferential: perpendicular to radial axis, change of radius

Radial: perpendicular to longitudinal axis, transmural from epi- to endocardium

In these axes, myocardial strain and strain rate can be determined. In systole, longitudinal and circumferential strains are negative because of fiber shortening (spatial approximation of speckles), whereas radial strain gets positive due to myocardial thickening (increasing spatial distance of corresponding speckles in the radial direction) [10,21]. 

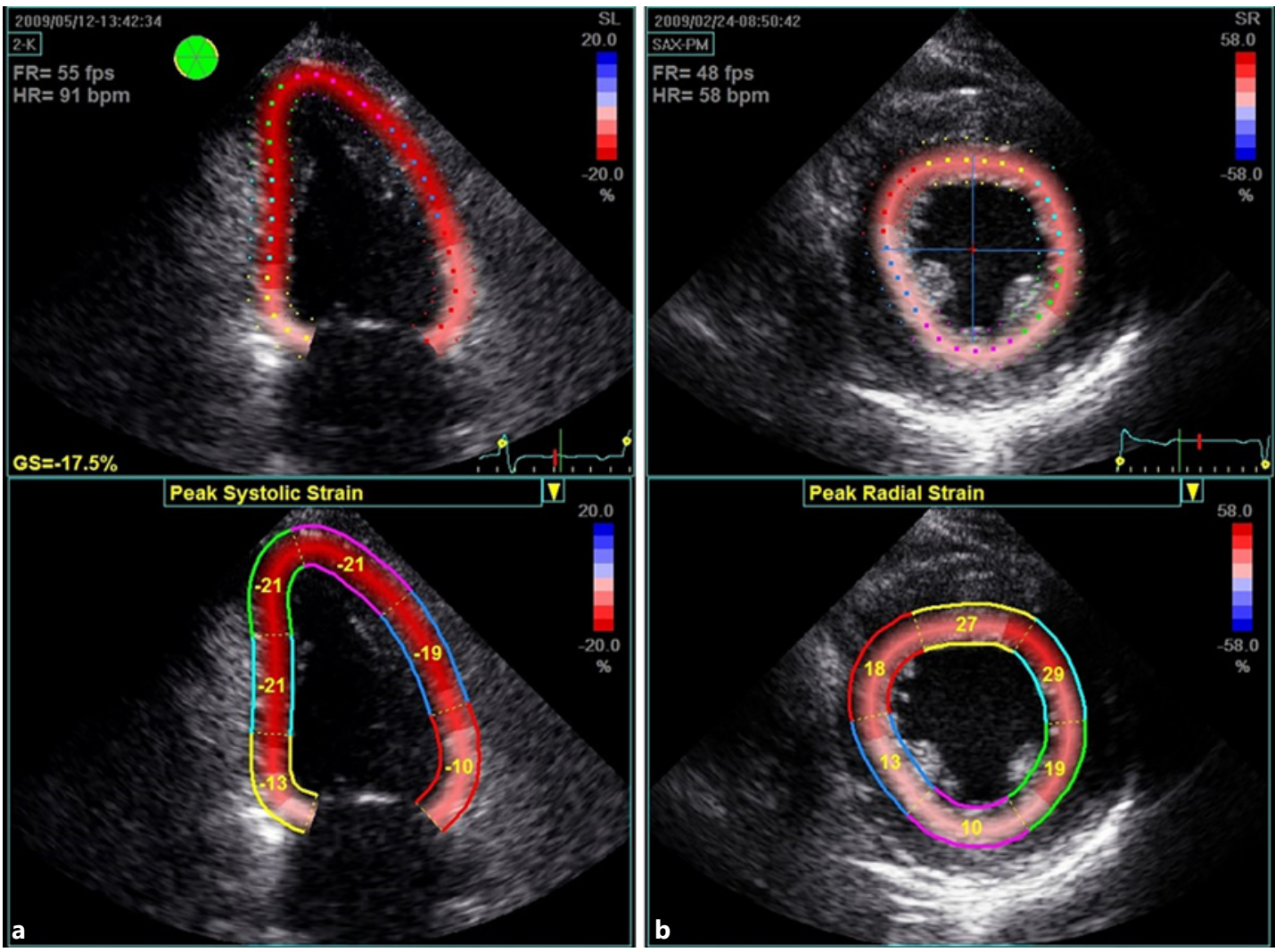

Fig. 2. Definition of endo- and epicardial borders by speckles in apical 2-chamber view (a) and short axis view at midpapillary level (b) and processed strain values per each segment.

We acquired the following left ventricular strain values and strain rates with the peak values during the entire heart cycle over the entire cardiac wall. One average value of 6 regional segments was acquired for longitudinal strain (SL peak G), early diastolic strain rate longitudinal (SrL peak E) and late diastolic strain rate longitudinal (SrL peak A) in 4-chamber, 2-chamber, and long-axis views. Similarly, 1 average value was obtained for circumferential strain and radial strain (SC peak G and SR peak G), early diastolic strain rate circumferential and radial (SrC peak E and SrR peak E), and late diastolic strain rate circumferential and radial ( $\mathrm{SrC}$ peak A and SrR peak A) of the parasternal short-axis view at the midpapillary level, respectively. One global longitudinal value could further be calculated by averaging the 3 values obtained from each apical view (SL peak G global [i.e., global SL], SrL peak E global [i.e., global early diastolic strain rate longitudinal], SrL peak A global [i.e., global late diastolic strain rate longitudinal]). Global values for circumferential and radial strains could not be calculated as echocardiographic data for basal and apical levels were partly incomplete.

Tracking quality of each segment was ensured as the software package consistently generates a quality scale ranging from 1.0 (optimal) to 3.0 (inadequate). As in former studies, only analyzed segments with tracking quality $\leq 2.0$ were evaluated [11, 22, 23]. Patients' outcome data were not apparent for the investigator.

Furthermore, intra- and interobserver variabilities were calculated from 10 measurements of 1 patient's strain values performed by 1 rater and of 2 raters' assessing strain values of the same patients, respectively. Intraobserver variability was $1.54 \%$, interobserver variability was $6.49 \%$. 
Table 1. Clinical characteristics of all patients stratified according to survivors, non-survivors, and CV death

\begin{tabular}{|c|c|c|c|c|c|}
\hline Characteristic & $\begin{array}{l}\text { Survivors } \\
(n=179)\end{array}$ & $\begin{array}{l}\text { Non-survivors } \\
(n=106)\end{array}$ & $p$ value $^{1}$ & $\begin{array}{l}\text { CV death } \\
(n=35)\end{array}$ & $p$ value $^{1}$ \\
\hline \multicolumn{6}{|l|}{ General demographics } \\
\hline Men & $106(59)$ & $75(71)$ & 0.0567 & $26(74)$ & 0.1909 \\
\hline Age, years & $69.51 \pm 11.57$ & $74.55 \pm 10.19$ & 0.0002 & $75.20 \pm 9.70$ & 0.0191 \\
\hline Height, $\mathrm{cm}$ & $168.97 \pm 9.09$ & $170.32 \pm 8.59$ & 0.2328 & $170.00 \pm 8.84$ & 0.7228 \\
\hline Body weight, kg & $79.30 \pm 16.78$ & $78.33 \pm 14.11$ & 0.6184 & $76.22 \pm 14.20$ & 0.262 \\
\hline $\mathrm{BMI}, \mathrm{kg} / \mathrm{m}^{2}$ & $27.91 \pm 10.34$ & $26.98 \pm 9.02$ & 0.1766 & $26.29 \pm 8.36$ & 0.1483 \\
\hline Current or previous smokers & $87(49)$ & $56(53)$ & 0.5405 & $22(63)$ & 0.1481 \\
\hline Systolic blood pressure, mm Hg & $128.94 \pm 39.16$ & $113.32 \pm 35.86$ & 0.0109 & $106.32 \pm 38.85$ & 0.0356 \\
\hline Diastolic blood pressure, $\mathrm{mm} \mathrm{Hg}$ & $69.39 \pm 19.77$ & $63.67 \pm 20.31$ & 0.0862 & $61.82 \pm 23.01$ & 0.2286 \\
\hline $\mathrm{LVEF}, \%$ & $48.53 \pm 12.75$ & $42.29 \pm 13.96$ & 0.0002 & $38.85 \pm 13.32$ & 0.0011 \\
\hline \multicolumn{6}{|l|}{ Previous diagnoses } \\
\hline Hypertension & $154(86)$ & $89(84)$ & 0.7298 & $30(86)$ & 1 \\
\hline Coronary artery disease & $103(58)$ & $77(73)$ & 0.0113 & $26(74)$ & 0.1902 \\
\hline STEMI & $14(8)$ & $5(5)$ & 0.4616 & $3(9)$ & 0.7141 \\
\hline NSTEMI & $76(42)$ & $64(60)$ & 0.0047 & $22(63)$ & 0.1041 \\
\hline Coronary stent & $49(27)$ & $35(33)$ & 0.3475 & $13(37)$ & 0.3234 \\
\hline Coronary artery bypass & $27(15)$ & $31(29)$ & 0.0059 & $11(31)$ & 0.1137 \\
\hline Peripheral artery occlusive disease & $24(13)$ & $26(25)$ & 0.0235 & $11(31)$ & 0.031 \\
\hline Prior stroke & $29(16)$ & $28(26)$ & 0.0462 & $13(37)$ & 0.0118 \\
\hline Valvular heart disease & $145(81)$ & $89(84)$ & 0.632 & $30(86)$ & 0.6449 \\
\hline Congestive heart failure & $111(62)$ & $70(66)$ & 0.1605 & $20(57)$ & 0.7502 \\
\hline Endocarditis & $5(3)$ & $2(2)$ & 1 & $1(3)$ & 1 \\
\hline Myocarditis & $1(1)$ & $0(0)$ & 1 & $0(0)$ & 1 \\
\hline Pericarditis & $1(1)$ & $0(0)$ & 1 & $0(0)$ & 1 \\
\hline Atrial fibrillation & $64(36)$ & $59(56)$ & 0.0013 & $16(46)$ & 0.8557 \\
\hline Pacemaker & $55(31)$ & $43(41)$ & 0.0954 & $17(49)$ & 0.0858 \\
\hline Cardiomyopathy & $48(27)$ & $45(42)$ & 0.0088 & $16(46)$ & 0.0857 \\
\hline Diabetes mellitus type 1 or 2 & $61(34)$ & $42(40)$ & 0.3732 & $16(46)$ & 0.2595 \\
\hline Coronary angiography and intervention & $36(20)$ & $30(28)$ & 0.1459 & $13(37)$ & 0.0521 \\
\hline Renal anemia & $20(11)$ & $12(11)$ & 1 & $7(20)$ & 0.0888 \\
\hline Hyperuricemia & $50(28)$ & $30(28)$ & 1 & $10(29)$ & 1 \\
\hline Parathyroidectomy & $0(0)$ & $0(0)$ & & $0(0)$ & \\
\hline Secondary hyperparathyroidism & $17(9)$ & $4(4)$ & 0.0997 & $4(11)$ & 0.3052 \\
\hline Diabetic nephropathy & $21(12)$ & $11(10)$ & 0.8468 & $4(11)$ & 1 \\
\hline Nephrosclerosis & $7(4)$ & $3(3)$ & 0.7489 & $2(6)$ & 0.3533 \\
\hline Polycystic kidney disease & $2(1)$ & $0(0)$ & 0.5312 & $0(0)$ & 1 \\
\hline Interstitial nephritis & $0(0)$ & $0(0)$ & & $0(0)$ & \\
\hline Glomerulonephritis & $8(4)$ & $1(1)$ & 0.1608 & $0(0)$ & 0.6069 \\
\hline Nephrectomy & $7(4)$ & $5(5)$ & 0.7663 & $0(0)$ & 0.3724 \\
\hline \multicolumn{6}{|l|}{ Medications } \\
\hline ACE inhibitor & $113(63)$ & $59(56)$ & 0.2595 & $15(43)$ & 0.0275 \\
\hline$\beta$-Blocker & $147(82)$ & $87(82)$ & 1 & 31 (89) & 0.3533 \\
\hline Amiodarone & $18(10)$ & $15(14)$ & 0.3396 & $6(17)$ & 0.2645 \\
\hline Angiotension II antagonist & $41(23)$ & $24(23)$ & 1 & $9(26)$ & 0.6693 \\
\hline Calcium channel blocker & $20(11)$ & $12(11)$ & 1 & $4(11)$ & 1 \\
\hline Sympatholytics & $16(9)$ & $9(8)$ & 1 & $4(11)$ & 0.5259 \\
\hline Diuretic & $153(85)$ & $96(91)$ & 0.2689 & $31(89)$ & 1 \\
\hline Cinacalcet & $2(1)$ & $0(0)$ & 0.5312 & $0(0)$ & 1 \\
\hline Phosphate binder, calcium-containing & $1(1)$ & $2(2)$ & 0.5577 & $0(0)$ & 1 \\
\hline Phosphate binder, non-calcium-containing & $3(2)$ & $0(0)$ & 0.2967 & $0(0)$ & 1 \\
\hline Phenprocoumon & $64(36)$ & $42(40)$ & 0.5283 & $17(49)$ & 0.1407 \\
\hline Erythropoietin & $12(7)$ & $8(8)$ & 0.8132 & $4(11)$ & 0.2854 \\
\hline Vitamin D & 31 (17) & $14(13)$ & 0.4037 & $7(20)$ & 0.4611 \\
\hline \multicolumn{6}{|l|}{ Laboratory parameters } \\
\hline Sodium, $\mathrm{mmol} / \mathrm{L}$ & $140.08 \pm 3.56$ & $140.64 \pm 4.25$ & 0.236 & $140.74 \pm 4.73$ & 0.4579 \\
\hline Potassium, mmol/L & $4.36 \pm 0.56$ & $4.33 \pm 0.61$ & 0.6665 & $4.38 \pm 0.59$ & 0.7348 \\
\hline Calcium, mmol/L & $2.22 \pm 0.17$ & $2.18 \pm 0.17$ & 0.0445 & $2.17 \pm 0.16$ & 0.1561 \\
\hline
\end{tabular}




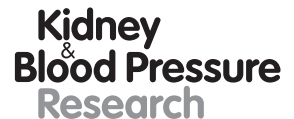

\begin{tabular}{l|l}
\hline \multicolumn{2}{l}{ Kidney Blood Press Res 2019;44:690-703 } \\
\hline DOI: 10.1159/000501225 & $\begin{array}{l}\text { @ 2019 The Author(s). Published by S. Karger AG, Basel } \\
\text { www.karger.com/kbr }\end{array}$ \\
\hline
\end{tabular}

Jahn et al.: Strain and Mortality in CKD Patients

Table 1 (continued)

\begin{tabular}{|c|c|c|c|c|c|}
\hline Characteristic & $\begin{array}{l}\text { Survivors } \\
(n=179)\end{array}$ & $\begin{array}{l}\text { Non-survivors } \\
(n=106)\end{array}$ & $p$ value $^{1}$ & $\begin{array}{l}\text { CV death } \\
(n=35)\end{array}$ & $p$ value $^{1}$ \\
\hline Creatinine, mg/dL & $1.75 \pm 0.59$ & $1.85 \pm 0.60$ & 0.1709 & $1.97 \pm 0.71$ & 0.1112 \\
\hline $\mathrm{eGFR}, \mathrm{mL} / \mathrm{min} / 1.73 \mathrm{~m}^{2}$ & $38.65 \pm 12.17$ & $35.97 \pm 11.89$ & 0.07 & $34.52 \pm 12.39$ & 0.1158 \\
\hline Protein, g/L & $67.96 \pm 8.79$ & $67.35 \pm 10.45$ & 0.6221 & $66.79 \pm 7.62$ & 0.4653 \\
\hline $\mathrm{CRP}, \mathrm{mg} / \mathrm{L}$ & $22.51 \pm 28.47$ & $26.09 \pm 39.01$ & 0.3806 & $23.56 \pm 27.34$ & 0.9521 \\
\hline Phosphate, mmol/L & $1.19 \pm 0.33$ & $1.25 \pm 0.33$ & 0.3121 & $1.33 \pm 0.46$ & 0.1221 \\
\hline Triglycerides, mg/dL & $162.39 \pm 97.39$ & $153.92 \pm 100.11$ & 0.5594 & $139.67 \pm 73.79$ & 0.1918 \\
\hline Cholesterol, mg/dL & $178.62 \pm 55.11$ & $163.59 \pm 68.10$ & 0.0746 & $147.62 \pm 65.06$ & 0.0395 \\
\hline Hemoglobin, g/L & $121.73 \pm 20.38$ & $117.62 \pm 22.20$ & 0.1214 & $116.91 \pm 19.21$ & 0.2928 \\
\hline Albumin, g/L & $35.92 \pm 8.86$ & $31.63 \pm 8.64$ & 0.0878 & $32.86 \pm 6.86$ & 0.6455 \\
\hline CKD stage 3 & 127 (71) & $66(62)$ & 0.1497 & $19(54)$ & 0.0828 \\
\hline
\end{tabular}

${ }^{1}$ Versus survivors, Fisher's exact test.

Data are $n(\%)$ or mean \pm SD.

LVEF, left ventricular ejection fraction; STEMI, ST-elevation myocardial infarction; NSTEMI, non-STEMI; ACE, angiotensinconverting enzyme; eGFR, estimated glomerular filtration rate; CRP, C-reactive protein; CKD, chronic kidney disease; CV, cardiovascular; BMI, body mass index.

\section{Follow-Up}

CV death (death attributed to myocardial infarction, cardiogenic shock or stroke) within 2.5 years was determined as first target outcome. All-cause mortality within 2.5 years was defined as the second target outcome. Follow-up data within 2.5 years after echocardiography were collected by review of the patients' hospital chart. In case of incomplete data, we contacted the patient, the patient's close relatives or general practitioner via phone. The reviewers investigating the outcomes were blinded to the patients' echocardiographic information.

\section{Statistical Analysis}

All statistical analyses were assessed with SAS software version 9.4 (SAS Institute Inc., Cary, NC, USA) using Windows 10 . Continuous variables were expressed as mean \pm SD; categorical variables as absolute frequencies and percentages.

For comparison between 2 groups, the unpaired $t$ test was performed for continuous variables, likewise $\chi^{2}$ and Fisher's exact tests were performed for categorical variables. Cox proportional hazards regression was used to investigate the influence of global SL on CV and all-cause mortality. Additionally, we adjusted in a multivariate Cox model for sex, age, diabetes type 1 and 2, eGFR (CKD Epidemiology Collaboration), and preexisting CV disease (i.e., coronary artery disease, STEMI, NSTEMI, stroke) to find the impact of global SL in relation to potential confounders. The Kaplan-Meier method provided estimations about cumulative survival. By using log-rank test, comparisons between survival curves could be observed. ROC analysis was implemented to calculate optimal cut-off values. The best cut-off value was determined by using the Youden index. Differences were considered statistically significant if $p$ values were $<0.05$.

\section{Results}

Clinical Characteristics and Strain Parameters

The mean age was $71 \pm 11$ years in the CKD group and $69 \pm 9$ years in the control group, respectively. In the CKD group, 181 (64\%) patients and in the control group $23(68 \%)$ were 
Table 2. Strain parameters of CKD patients in stage 3 or 4 compared to the healthy control group

\begin{tabular}{lccc}
\hline Strain parameter, \% & $\begin{array}{l}\text { CKD patients } \\
(n=285)\end{array}$ & $\begin{array}{l}\text { Control group } \\
(n=34)\end{array}$ & $p$ value \\
\hline SL peak G global & $-11.41 \pm 4.31$ & $-13.59 \pm 3.91$ & 0.0040 \\
SrL peak E global & $0.83 \pm 0.32$ & $1.03 \pm 0.26$ & 0.0003 \\
SrL peak A global & $0.74 \pm 0.39$ & $0.90 \pm 0.34$ & 0.0163 \\
SC peak G & $-13.64 \pm 6.03$ & $-14.53 \pm 5.77$ & 0.4429 \\
SrC peak E & $1.43 \pm 0.6$ & $1.43 \pm 0.52$ & 0.9387 \\
SrC peak A & $0.89 \pm 0.52$ & $0.98 \pm 0.48$ & 0.3415 \\
SR peak G & $18.15 \pm 15.11$ & $25.97 \pm 20.55$ & 0.0125 \\
SrR peak E & $-1.52 \pm 0.95$ & $-1.46 \pm 0.92$ & 0.7348 \\
SrR peak A & $-1.1 \pm 0.99$ & $1.58 \pm 2.72$ & 0.0605 \\
\hline
\end{tabular}

SL peak G global, global longitudinal strain; SrL peak E global, global early diastolic strain rate longitudinal; SrL peak A global, global late diastolic strain rate longitudinal; SC peak G, circumferential strain; SrC peak E, early diastolic strain rate circumferential; SrC peak A, late diastolic strain rate circumferential; SR peak G, radial strain; SrR peak E, early diastolic strain rate radial; SrR peak A, late diastolic strain rate radial; CKD, chronic kidney disease.

Table 3. Comparison between CKD patients in stages 3 and 4

\begin{tabular}{lccc}
\hline Strain parameter, \% & $\begin{array}{l}\text { CKD patients in stage } 3 \\
(n=193)\end{array}$ & $\begin{array}{l}\text { CKD patients in stage } 4 \\
(n=92)\end{array}$ & $p$ value \\
\hline SL peak G global & $-11.15 \pm 4.44$ & $-11.96 \pm 3.98$ & 0.125 \\
SrL peak E global & $0.83 \pm 0.34$ & $0.84 \pm 0.29$ & 0.6961 \\
SrL peak A global & $0.72 \pm 0.39$ & $0.8 \pm 0.4$ & 0.1149 \\
SC peak G & $-13.55 \pm 6.01$ & $-13.84 \pm 6.12$ & 0.7406 \\
SrC peak E & $1.41 \pm 0.59$ & $1.46 \pm 0.63$ & 0.5617 \\
SrC peak A & $0.87 \pm 0.52$ & $0.94 \pm 0.53$ & 0.3525 \\
SR peak G & $18.18 \pm 15.6$ & $18.07 \pm 14.05$ & 0.9567 \\
SrR peak E & $-1.52 \pm 1$ & $-1.52 \pm 0.82$ & 0.9974 \\
SrR peak A & $-1.06 \pm 1$ & $-1.18 \pm 0.95$ & 0.3817 \\
\hline
\end{tabular}

SL peak G global, global longitudinal strain; SrL peak E global, global early diastolic strain rate longitudinal; SrL peak A global, global late diastolic strain rate longitudinal; SC peak G, circumferential strain; SrC peak E, early diastolic strain rate circumferential; SrC peak A, late diastolic strain rate circumferential; SR peak G, radial strain; SrR peak E, early diastolic strain rate radial; SrR peak A, late diastolic strain rate radial; CKD, chronic kidney disease.

male. At the time of the examination, 193 (68\%) patients were in CKD stages 3 and 92 (32\%) in stage 4 . The mean LVEF was $46 \pm 14 \%$. A total of 181 patients (64\%) suffered from heart failure and $234(82 \%)$ from valvular disease, even though predominantly in a non-severe status. The clinical characteristics of all patients are summarized in Table 1.

In the CKD group, all measured longitudinal and radial strain values were significantly reduced (i.e., less negative) compared to the control group (Table 2). A total of 243 (85\%) of the CKD patients had reduced global SL values. There were no significant differences concerning strain values between CKD 3 and 4 patients (Table 3). SL peak G global, SrL peak E global, SrL peak A global, SC peak G, SR peak G, and SrR peak E were significantly decreased in patients who died of CV disease during the follow-up period (Table 4) when compared to 
Table 4. Comparison between CKD patients who died of CV disease within the follow-up period and survivors

\begin{tabular}{lccc}
\hline Strain parameter, \% & $\begin{array}{l}\text { CKD patients CV death } \\
(n=35)\end{array}$ & $\begin{array}{l}\text { CKD patients no CV death } \\
(n=250)\end{array}$ & $p$ value \\
\hline SL peak G global & $-9.38 \pm 3.99$ & $-11.7 \pm 4.28$ & 0.0026 \\
SrL peak E global & $0.71 \pm 0.32$ & $0.85 \pm 0.32$ & 0.0196 \\
SrL peak A global & $0.57 \pm 0.5$ & $0.77 \pm 0.37$ & 0.0044 \\
SC peak G & $-10.51 \pm 5.69$ & $-14.11 \pm 5.96$ & 0.0027 \\
SrC peak E & $1.25 \pm 0.65$ & $1.45 \pm 0.59$ & 0.1232 \\
SrC peak A & $0.81 \pm 0.44$ & $0.9 \pm 0.53$ & 0.2982 \\
SR peak G & $11.05 \pm 13.06$ & $19.21 \pm 15.13$ & 0.0033 \\
SrR peak E & $-1.19 \pm 1.19$ & $-1.57 \pm 0.9$ & 0.0401 \\
SrR peak A & $-0.85 \pm 1.12$ & $-1.14 \pm 0.96$ & 0.1945 \\
\hline
\end{tabular}

SL peak G global, global longitudinal strain; SrL peak E global, global early diastolic strain rate longitudinal; SrL peak A global, global late diastolic strain rate longitudinal; SC peak G, circumferential strain; SrC peak E, early diastolic strain rate circumferential; SrC peak A, late diastolic strain rate circumferential; SR peak G, radial strain; SrR peak E, early diastolic strain rate radial; SrR peak A, late diastolic strain rate radial; CKD, chronic kidney disease; CV, cardiovascular.

Table 5. Comparison between CKD patients who died of any reason within the follow-up period and survivors

\begin{tabular}{lccc}
\hline Strain parameter, $\%$ & $\begin{array}{l}\text { CKD patients } \\
\text { non-survivors } \\
(n=106)\end{array}$ & $\begin{array}{l}\text { CKD patients } \\
\text { survivors } \\
(n=179)\end{array}$ & $p$ value \\
\hline SL peak G global & $-10.22 \pm 4.29$ & $-12.12 \pm 4.17$ & 0.0003 \\
SrL peak E global & $0.78 \pm 0.34$ & $0.86 \pm 0.31$ & 0.0384 \\
SrL peak A global & $0.66 \pm 0.43$ & $0.79 \pm 0.37$ & 0.0121 \\
SC peak G & $-11.51 \pm 5.43$ & $-14.77 \pm 6.05$ & 0.0001 \\
SrC peak E & $1.31 \pm 0.64$ & $1.49 \pm 0.57$ & 0.1348 \\
SrC peak A & $0.82 \pm 0.48$ & $0.93 \pm 0.54$ & 0.0013 \\
SR peak G & $13.78 \pm 11.82$ & $20.46 \pm 16.15$ & 0.0333 \\
SrR peak E & $-1.34 \pm 1.01$ & $-1.62 \pm 0.90$ & 0.0162 \\
SrR peak A & $-0.9 \pm 0.87$ & $-1.21 \pm 1.03$ & $<$ \\
\hline
\end{tabular}

SL peak G global, global longitudinal strain; SrL peak E global, global early diastolic strain rate longitudinal; SrL peak A global, global late diastolic strain rate longitudinal; SC peak G, circumferential strain; SrC peak E, early diastolic strain rate circumferential; SrC peak A, late diastolic strain rate circumferential; SR peak G, radial strain; SrR peak E, early diastolic strain rate radial; SrR peak A, late diastolic strain rate radial; CKD, chronic kidney disease.

values of survivors. In addition, more strain values differed significantly between surviving patients and patients who died of any reason (Table 5).

\section{Outcome Parameters}

In a period of 2.5 years, 35 CKD patients (12\%) died of CV disease, after a mean follow-up of $338 \pm 294$ days. All-cause mortality during this period was 37\% (106 of 285 patients). Septicemia (14 patients [5\%]), cancer (12 patients [4\%]), others (14 patients [5\%]) represented other causes of death. For 31 patients $(11 \%)$, the reasons for death remained unknown. Patients were categorized according to survival and death and presented in Table 1 with corresponding patient characteristics. 


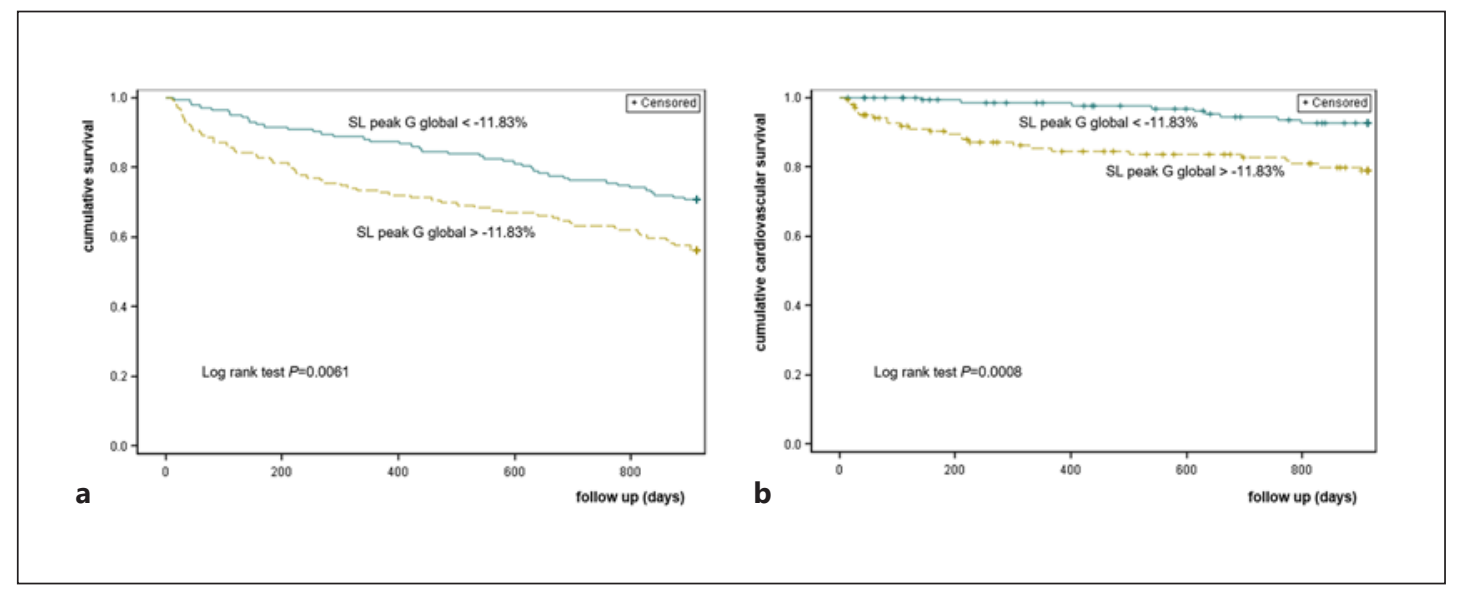

Fig. 3. Kaplan-Meier survival analyses and log-rank test show that less negative strain values for CKD patients at stages 3 and 4 result in a poorer outcome for both all-cause mortality (a) and CV mortality (b). $-11.83 \%$ represents the median of all assessed global SL values. SL peak G global, global longitudinal strain. SL, longitudinal strain; CV; cardiovascular.

Table 6. Univariate Cox models for global longitudinal strain as predictor of all-cause and CV mortalities

\begin{tabular}{lccc}
\hline Parameter & $p$ value & HR & $95 \%$ HR CI \\
\hline $\begin{array}{l}\text { Cox univariate - all-cause mortality } \\
\text { Global longitudinal strain }\end{array}$ & 0.0003 & 1.09 & $1.04-1.14$ \\
\hline $\begin{array}{l}\text { Cox univariate- CV mortality } \\
\text { Global longitudinal strain }\end{array}$ & 0.0008 & 1.15 & $1.06-1.25$ \\
\hline
\end{tabular}

Global longitudinal strain, average of longitudinal strain of 4-chamber, 2-chamber, and long-axis view; CV, cardiovascular; HR, hazard ratio.

Stratified into 2 groups according to the median global SL $(-11.8 \%)$, the differences of cumulative survival between CKD patients with better strain values (more negative) and reduced strain values (less negative) became apparent in Kaplan-Meier survival analyses. By means of log-rank tests, a significant difference between these groups was found both concerning CV mortality (log-rank test: $p=0.0008$ ) and all-cause mortality (log-rank test: $p=0.006$ ), which confirms the assumption that reduced global SL is associated with both increased CV and all-cause mortality (Fig. 3).

Next, we used global SL, which is believed to be more sensitive in identifying myocardial disease and more useful in clinical practice compared to circumferential and radial strains [24], to investigate risk factors for mortality. Univariate Cox regression was performed and showed global SL to be a predictor of CV mortality (hazard ratio [HR] 1.15, 95\% CI 1.06-1.25; $p=0.0008$ ) and all-cause mortality (HR 1.09, 95\% CI 1.04-1.14; $p=0.0003$; Table 6). Additionally, potential confounders (i.e., sex, age, diabetes type 1 or 2, history of CV events [i.e., coronary artery disease, STEMI, NSTEMI, stroke], GFR) were included in a multivariate Cox regression analysis. Global SL remained an independent risk factor of CV mortality (HR 1.16, 95\% CI 1.06-1.27; $p=0.0019$ ) and all-cause mortality (HR 1.08, 95\% CI 1.03-1.14; $p=0.0026$ ); furthermore, age (HR 1.04, 95\% CI 1.02-1.06; $p=0.0009$ ) and eGFR (HR 0.98, 95\% CI 0.96-1; $p=0.0124$ ) were independent predictors (Table 7). ROC analysis was also implemented for 
Table 7. Multivariate Cox models for predictors of all-cause and CV mortalities

\begin{tabular}{lccc}
\hline Parameter & $p$ value & HR & HR 95\% CI \\
\hline Cox multivariate - all-cause mortality & & & \\
Global longitudinal strain, \% & 0.0026 & 1.08 & $1.03-1.14$ \\
Gender & 0.1080 & 0.68 & $0.43-1.09$ \\
Age, years & 0.0009 & 1.04 & $1.02-1.06$ \\
Diabetes 1 or 2 & 0.2356 & 0.78 & $0.51-1.18$ \\
CV diagnoses & 0.2173 & 0.68 & $0.37-1.26$ \\
eGFR, mL/min/1.73 m ${ }^{2}$ & 0.0124 & 0.98 & $0.96-1.0$ \\
\hline Cox multivariate - CV mortality & & & \\
Global longitudinal strain, \% & 0.0019 & 1.16 & $1.06-1.27$ \\
Gender & 0.1196 & 0.51 & $0.22-1.19$ \\
Age, years & 0.0204 & 1.05 & $1.01-1.1$ \\
Diabetes 1 or 2 & 0.0885 & 0.54 & $0.27-1.1$ \\
CV diagnoses & 0.6782 & 0.8 & $0.27-2.35$ \\
eGFR, mL/min/1.73 m $^{2}$ & 0.0176 & 0.97 & $0.94-0.99$
\end{tabular}

Global longitudinal strain, average of longitudinal strain of 4-chamber, 2-chamber, and long-axis view; eGFR, estimated glomerular filtration rate; CV, cardiovascular; HR, hazard ratio.

Table 8. ROC analysis

\begin{tabular}{lllrrc}
\hline Parameter & $\begin{array}{l}\text { Area under } \\
\text { the ROC curve }\end{array}$ & $\begin{array}{l}\text { Youden } \\
\text { index }\end{array}$ & $\begin{array}{l}\text { Cut-off } \\
\text { value, } \%\end{array}$ & Sensitivity & Specificity \\
\hline $\begin{array}{l}\text { ROC analysis - all-cause mortality } \\
\text { Global longitudinal strain }\end{array}$ & 0.63 & 0.21 & -9.26 & 0.45 & 0.76 \\
LVEF & 0.63 & 0.23 & 51 & 0.7 & 0.54 \\
\hline $\begin{array}{l}\text { ROC analysis - CV mortality } \\
\text { Global longitudinal strain }\end{array}$ & 0.66 & 0.3 & -12.42 & 0.83 & 0.47 \\
LVEF & 0.68 & 0.33 & 50 & 0.83 & 0.5 \\
\hline
\end{tabular}

Global longitudinal strain, average of longitudinal strain of 4-chamber, 2-chamber and long-axis view; $\mathrm{CV}$, cardiovascular; LVEF, left ventricular ejection fraction.

survival analyses. The area under the ROC curve of global SL was 0.63 with a cut-off value of $-9.26 \%$ for prediction of all-cause mortality and 0.66 with a cut-off value of $-12.42 \%$ for prediction of CV mortality. Considering LVEF, the parameter predicted all-cause mortality in the same manner as global SL (area under the ROC curve 0.63 ; cut-off value $51 \%$ ). Concerning CV death, the area under the ROC curve of LVEF with a cut-off value of $50 \%$ and of global SL differed slightly (area under the ROC curve LVEF 0.68; area under the ROC curve global SL 0.66; Table 8).

\section{Discussion}

It is generally accepted that CKD accelerates the development of CV disease and increases mortality. According to the updated recommendations of the Kidney Disease Outcomes Quality Initiative CKD guidelines, CKD patients should be treated as a highest risk [25] group 


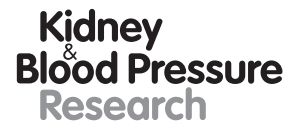

\begin{tabular}{l|l}
\hline Kidney Blood Press Res 2019;44:690-703 \\
\hline DOI: 10.1159/000501225 & $\begin{array}{l}\text { @ 2019 The Author(s). Published by S. Karger AG, Basel } \\
\text { www.karger.com/kbr }\end{array}$ \\
\hline
\end{tabular}

Jahn et al.: Strain and Mortality in CKD Patients

for CV disease, regardless of the presence of typical CV risk factors [25, 26]. The investigation of causes of CV impairment in patients with CKD is the subject of current research and abundant explanations of complex pathomechanisms already exist $[3,27,28]$. If diagnosed early, preventative strategies and treatments could be applied to prevent, for example, progressive heart failure or sudden cardiac death, that is, frequent reasons for CV death in ESRD [29].

STE, a novel echocardiographic technique, offers the opportunity to determine global and regional cardiac function in a non-invasive way. STE is easily utilizable, broadly available, and shows higher reproducibility than tissue Doppler imaging-derived strain modality [30, 31]. STE delivers important information about the severity of myocardial damage and allows predictions concerning left ventricular remodeling, outcomes after infarction, cardiac surgery or acute rejection in cardiac transplantation [22,32-36]. The technique also proved to be a useful indicator of myocardial involvement in diabetes, arterial hypertension and hypertrophy or valvular disease [37-40].

Our first major finding was that STE yielded several reduced values, in particular SL values, in patients with CKD 3-4 compared to a healthy control group. This is in line with prior studies, which analyzed STE at different stages of CKD and in dialysis patients [13-15].

The second major finding of our study was that impaired global SL predicted CV and allcause mortality in our patient group. We recently found global SL to be an independent outcome predictor in dialysis patients, likely because of its capability to detect myocardial hypertrophy and interstitial fibrosis, both of which characterize uremic cardiomyopathy [11]. Krishnasamy et al. [18] also reported global SL to be an independent predictor of allcause mortality. However, their patients were in CKD stages 1-3, and CV mortality was not assessed in their study. In a more recent study, the same authors confirmed global SL as an important predictor of all-cause and CV mortality and showed superiority of statistical power to LVEF [19]. However, that study focused on patients in CKD stages 4 and 5, including a high percentage of patients receiving dialysis (63\%). In another study, Panoulas et al. [17] involved all non-dialysis stages of CKD patients without CV disease. They established that certain longitudinal and circumferential strain values assessed with STE detected systolic and diastolic subtle aberrations in cardiac function, which was associated with an increased rate of CV events [17]. Thus, our study is the first assessing the predictive value of global SL in CKD patients with moderate CKD.

Several studies focused on the differences between global SL and conventional LVEF to analyze cardiac dysfunction. Some of these concluded that CKD patients usually preserve LVEF within the normal range while strain values, especially global SL, deteriorate [41]. These studies reported that global SL showed superior predictive power for mortality in contrast to LVEF [19]. Possible explanations were that LVEF rather assesses radial contraction [42] or that LVEF is maintained by circumferential function [13], whereas global SL measures subendocardial longitudinal myocardial fibers, which are more susceptible to, for example, damage from ischemia and increased wall stress [43, 44]. However, we could not confirm the predictive advantage of global SL compared to LVEF.

Besides STE, tissue Doppler imaging can be used to quantify strain. However, STE is considered to be superior in various respects (complexity of analysis, angle and frame rate dependency, measurement of radial and circumferential strains in addition to SL) [21].

Our study has some limitations. While an average value over 18 segments (6 left ventricular walls each for apical 2-, 3- and 4-chamber views) could be calculated for SL and strain rate values, respectively, only the midpapillary level was available in a sufficient number of cases for the assessment of circumferential and radial strains. Consequently, strain and strain rate parameters of the whole left ventricle could differ from our results involving apical and basal levels as these two parameters were partly incomplete in our study. The 
association between strain values and patients' outcome cannot be generalized to other cohorts as our study cohort was old and had a number of pre-existing diseases.

In conclusion, this study shows that STE detects reduced left ventricular myocardial function and allows the prediction of CV and all-cause mortality in patients with CKD stages 3 and 4. Especially in view of its reliability and practical operability, STE is a promising tool for the prediction of mortality risk in CKD patients.

\section{Statement of Ethics}

The retrospective study was approved by the Ethical Committee of the RWTH Aachen University Hospital (EK167/11) and carried out according to the Declaration of Helsinki.

\section{Disclosure Statement}

The authors declare no conflicts of interest.

\section{References}

1 Go AS, Chertow GM, Fan D, McCulloch CE, Hsu CY. Chronic kidney disease and the risks of death, cardiovascular events, and hospitalization. N Engl J Med. 2004 Sep; 351(13): 1296-305.

2 Culleton BF, Larson MG, Wilson PW, Evans JC, Parfrey PS, Levy D. Cardiovascular disease and mortality in a community-based cohort with mild renal insufficiency. Kidney Int. 1999 Dec; 56(6): 2214-9.

3 Hage FG, Venkataraman R, Zoghbi GJ, Perry GJ, DeMattos AM, Iskandrian AE. The scope of coronary heart disease in patients with chronic kidney disease. J Am Coll Cardiol. 2009 Jun; 53(23): 2129-40.

4 Sosnov J, Lessard D, Goldberg RJ, Yarzebski J, Gore JM. Differential symptoms of acute myocardial infarction in patients with kidney disease: a community-wide perspective. Am J Kidney Dis. 2006 Mar; 47(3): 378-84.

5 Martin FL, McKie PM, Cataliotti A, Sangaralingham SJ, Korinek J, Huntley BK, et al. Experimental mild renal insufficiency mediates early cardiac apoptosis, fibrosis, and diastolic dysfunction: a kidney-heart connection. Am J Physiol Regul Integr Comp Physiol. 2012 Jan; 302(2):R292-9.

6 Amann K, Tyralla K. Cardiovascular changes in chronic renal failure-pathogenesis and therapy. Clin Nephrol. 2002 Jul; 58 Suppl 1:S62-72.

7 Leitman M, Lysyansky P, Sidenko S, Shir V, Peleg E, Binenbaum M, et al. Two-dimensional strain-a novel software for real-time quantitative echocardiographic assessment of myocardial function. J Am Soc Echocardiogr. 2004 Oct; 17(10): 1021-9.

8 Dandel M, Lehmkuhl H, Knosalla C, Suramelashvili N, Hetzer R. Strain and strain rate imaging by echocardiography - basic concepts and clinical applicability. Curr Cardiol Rev. 2009 May; 5(2): 133-48.

9 Reisner SA, Lysyansky P, Agmon Y, Mutlak D, Lessick J, Friedman Z. Global longitudinal strain: a novel index of left ventricular systolic function. J Am Soc Echocardiogr. 2004 Jun; 17(6): 630-3.

10 D’hooge J, Heimdal A, Jamal F, Kukulski T, Bijnens B, Rademakers F, et al. Regional strain and strain rate measurements by cardiac ultrasound: principles, implementation and limitations. Eur J Echocardiogr. 2000 Sep; 1(3): 154-70.

11 Kramann R, Erpenbeck J, Schneider RK, Röhl AB, Hein M, Brandenburg VM, et al. Speckle tracking echocardiography detects uremic cardiomyopathy early and predicts cardiovascular mortality in ESRD. J Am Soc Nephrol. 2014 Oct; 25(10): 2351-65.

12 Bosselmann H, Tonder N, Sölétormos G, Rossing K, Iversen K, Goetze JP, et al. Influence of renal impairment on myocardial function in outpatients with systolic heart failure: an echocardiographic and cardiac biomarker study. Int J Cardiol. 2014 Dec; 177(3): 942-8.

13 Hassanin N, Alkemary A. Early Detection of Subclinical Uremic Cardiomyopathy Using Two-Dimensional Speckle Tracking Echocardiography. Echocardiography. 2016 Apr; 33(4): 527-36.

14 Liu YW, Su CT, Huang YY, Yang CS, Huang JW, Yang MT, et al. Left ventricular systolic strain in chronic kidney disease and hemodialysis patients. Am J Nephrol. 2011; 33(1): 84-90.

15 Yan P, Li H, Hao C, Shi H, Gu Y, Huang G, et al. 2D-speckle tracking echocardiography contributes to early identification of impaired left ventricular myocardial function in patients with chronic kidney disease. Nephron Clin Pract. 2011; 118(3):c232-40.

16 Chinali M, Matteucci MC, Franceschini A, Doyon A, Pongiglione G, Rinelli G, et al. Advanced Parameters of Cardiac Mechanics in Children with CKD: The 4C Study. Clin J Am Soc Nephrol. 2015 Aug; 10(8): 1357-63. 
Jahn et al.: Strain and Mortality in CKD Patients

17 Panoulas VF, Sulemane S, Konstantinou K, Bratsas A, Elliott SJ, Dawson D, et al. Early detection of subclinical left ventricular myocardial dysfunction in patients with chronic kidney disease. Eur Heart J Cardiovasc Imaging. 2015 May; 16(5): 539-48.

18 Krishnasamy R, Isbel NM, Hawley CM, Pascoe EM, Leano R, Haluska BA, et al. The association between left ventricular global longitudinal strain, renal impairment and all-cause mortality. Nephrol Dial Transplant. 2014 Jun; 29(6): 1218-25.

19 Krishnasamy R, Isbel NM, Hawley CM, Pascoe EM, Burrage M, Leano R, et al. Left Ventricular Global Longitudinal Strain (GLS) Is a Superior Predictor of All-Cause and Cardiovascular Mortality When Compared to Ejection Fraction in Advanced Chronic Kidney Disease. PLoS One. 2015 May; 10(5):e0127044.

20 McMurray JJ, Adamopoulos S, Anker SD, Auricchio A, Böhm M, Dickstein K, et al.; ESC Committee for Practice Guidelines. ESC Guidelines for the diagnosis and treatment of acute and chronic heart failure 2012: The Task Force for the Diagnosis and Treatment of Acute and Chronic Heart Failure 2012 of the European Society of Cardiology. Developed in collaboration with the Heart Failure Association (HFA) of the ESC. Eur Heart J. 2012 Jul; 33(14): 1787-847.

21 Blessberger H, Binder T. NON-invasive imaging: Two dimensional speckle tracking echocardiography: basic principles. Heart. 2010 May; 96(9): 716-22.

22 Becker M, Lenzen A, Ocklenburg C, Stempel K, Kühl H, Neizel M, et al. Myocardial deformation imaging based on ultrasonic pixel tracking to identify reversible myocardial dysfunction. J Am Coll Cardiol. 2008 Apr; 51(15): 1473-81.

23 Becker M, Kramann R, Franke A, Breithardt OA, Heussen N, Knackstedt C, et al. Impact of left ventricular lead position in cardiac resynchronization therapy on left ventricular remodelling. A circumferential strain analysis based on 2D echocardiography. Eur Heart J. 2007 May; 28(10): 1211-20.

24 Mor-Avi V, Lang RM, Badano LP, Belohlavek M, Cardim NM, Derumeaux G, et al. Current and evolving echocardiographic techniques for the quantitative evaluation of cardiac mechanics: ASE/EAE consensus statement on methodology and indications endorsed by the Japanese Society of Echocardiography. Eur J Echocardiogr. 2011 Mar; 12(3): 167-205.

25 Weiner DE, Sarnak MJ. A decade after the KDOQI CKD guidelines: impact on the cardiovascular disease-CKD paradigm. Am J Kidney Dis. 2012 Nov; 60(5): 710-2.

26 Tonelli M, Wiebe N, Culleton B, House A, Rabbat C, Fok M, et al. Chronic kidney disease and mortality risk: a systematic review. J Am Soc Nephrol. 2006 Jul; 17(7): 2034-47.

27 Schlieper G, Hess K, Floege J, Marx N. The vulnerable patient with chronic kidney disease. Nephrol Dial Transplant. 2016 Mar; 31(3): 382-90.

28 Schlieper G, Schurgers L, Brandenburg V, Reutelingsperger C, Floege J. Vascular calcification in chronic kidney disease: an update. Nephrol Dial Transplant. 2016 Jan; 31(1): 31-9.

29 Zoccali C. Left ventricular systolic dysfunction: a sudden killer in end-stage renal disease patients. Hypertension. 2010 Aug; 56(2): 187-8.

30 Belghitia H, Brette S, Lafitte S, Reant P, Picard F, Serri K, et al. Automated function imaging: a new operatorindependent strain method for assessing left ventricular function. Arch Cardiovasc Dis. 2008 Mar; 101(3): 163-9.

31 Dandel M, Hetzer R. Echocardiographic strain and strain rate imaging-clinical applications. Int J Cardiol. 2009 Feb; 132(1): 11-24.

32 Becker M, Ocklenburg C, Altiok E, Füting A, Balzer J, Krombach G, et al. Impact of infarct transmurality on layerspecific impairment of myocardial function: a myocardial deformation imaging study. Eur Heart J. 2009 Jun; 30(12): 1467-76.

33 Park YH, Kang SJ, Song JK, Lee EY, Song JM, Kang DH, et al. Prognostic value of longitudinal strain after primary reperfusion therapy in patients with anterior-wall acute myocardial infarction. J Am Soc Echocardiogr. 2008 Mar; 21(3): 262-7.

34 Ersbøll M, Valeur N, Mogensen UM, Andersen MJ, Møller JE, Velazquez EJ, et al. Prediction of all-cause mortality and heart failure admissions from global left ventricular longitudinal strain in patients with acute myocardial infarction and preserved left ventricular ejection fraction. J Am Coll Cardiol. 2013 Jun; 61(23): 2365-73.

35 Ternacle J, Berry M, Alonso E, Kloeckner M, Couetil JP, Randé JL, et al. Incremental value of global longitudinal strain for predicting early outcome after cardiac surgery. Eur Heart J Cardiovasc Imaging. 2013 Jan; 14(1): 77-84.

36 Pieper GM, Shah A, Harmann L, Cooley BC, Ionova IA, Migrino RQ. Speckle-tracking 2-dimensional strain echocardiography: a new noninvasive imaging tool to evaluate acute rejection in cardiac transplantation. J Heart Lung Transplant. 2010 Sep; 29(9): 1039-46.

37 Ng AC, Delgado V, Bertini M, van der Meer RW, Rijzewijk LJ, Hooi Ewe S, et al. Myocardial steatosis and biventricular strain and strain rate imaging in patients with type 2 diabetes mellitus. Circulation. 2010 Dec; 122(24): 2538-44.

38 Richand V, Lafitte S, Reant P, Serri K, Lafitte M, Brette S, et al. An ultrasound speckle tracking (two-dimensional strain) analysis of myocardial deformation in professional soccer players compared with healthy subjects and hypertrophic cardiomyopathy. Am J Cardiol. 2007 Jul; 100(1): 128-32.

39 Marciniak A, Sutherland GR, Marciniak M, Claus P, Bijnens B, Jahangiri M. Myocardial deformation abnormalities in patients with aortic regurgitation: a strain rate imaging study. Eur J Echocardiogr. 2009 Jan; 10(1): 112-9. 
40 Blessberger H, Binder T. Two dimensional speckle tracking echocardiography: clinical applications. Heart. 2010 Dec; 96(24): 2032-40.

41 Edwards NC, Hirth A, Ferro CJ, Townend JN, Steeds RP. Subclinical abnormalities of left ventricular myocardial deformation in early-stage chronic kidney disease: the precursor of uremic cardiomyopathy? J Am Soc Echocardiogr. 2008 Dec; 21(12): 1293-8.

42 Buckberg G, Hoffman JI, Mahajan A, Saleh S, Coghlan C. Cardiac mechanics revisited: the relationship of cardiac architecture to ventricular function. Circulation. 2008 Dec; 118(24): 2571-87.

43 Reimer KA, Jennings RB. The "wavefront phenomenon" of myocardial ischemic cell death. II. Transmural progression of necrosis within the framework of ischemic bed size (myocardium at risk) and collateral flow. Lab Invest. 1979 Jun; 40(6): 633-44.

44 Algranati D, Kassab GS, Lanir Y. Why is the subendocardium more vulnerable to ischemia? A new paradigm. Am J Physiol Heart Circ Physiol. 2011 Mar; 300(3):H1090-100. 